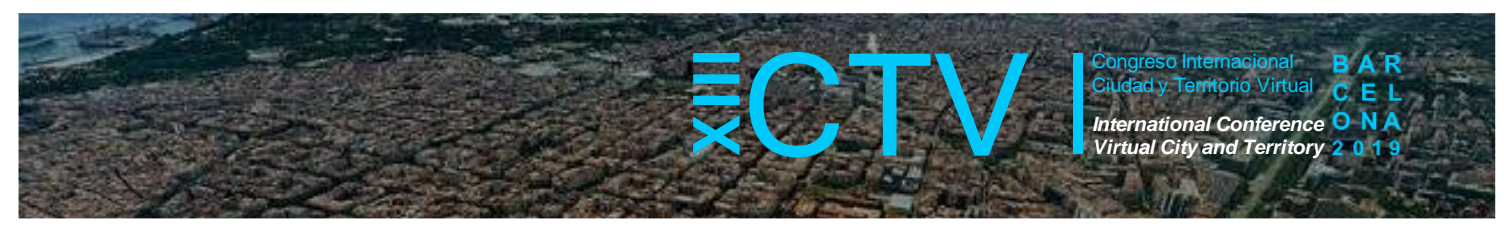

\title{
CENTRALIDAD Y SUBCENTRALIDAD EN CIUDADES CON BAJA REGULACIÓN, EL CASO ANTOFAGASTA Y LA SERENA EN CHILE
}

\author{
Aguirre-Núñez, Carlos ${ }^{1 *}$; Marmolejo-Duarte, Carlos ${ }^{2}$; Vergara Perucich, José Francisco ${ }^{3}$ \\ Remisión inicial: 2019-05-31; Remisión definitiva: 2019-10-13; Publicación: 2019-12-21 \\ Citación: Aguirre-Núñez, C. (2019). Centralidad y subcentralidad en ciudades con baja regulación, el caso \\ Antofagasta y la Serena en Chile. En XIII CTV 2019 Proceedings: XIII International Conference \\ on Virtual City and Territory: "Challenges and paradigms of the contemporary city": UPC, \\ Barcelona, October 2-4, 2019. Barcelona: CPSV, 2019, p. 8476. E-ISSN 2604-6512. DOI \\ http://dx.doi.org/10.5821/ctv.8476
}

\section{Resumen}

Este trabajo busca identificar subcentros laborales y de servicios en la zona, mediante la información de las encuestas de origen destino del ministerio de transporte chileno, y analizar la actividad edificatoria, y de mercado de vivienda en cada una de ellas Se seguirá la metodología de identificación, establecida en Marmolejo y Aguirre (2011) respecto a los peak de densidad y la utilización de la densidad de trabajadores, su versión de densidad compuesta y validando mediante una versión adecuada de la densidad tiempo.

Existe aún clara relación entre la aglomeración de usos y segregación en la ciudad y las bajas regulaciones en el mercado de suelo y vivienda. En Chile, existe una baja regulación de los mercados de vivienda y en particular ambas ciudades cuentan con regulaciones a nivel municipal, que, dado su complejidad de aprobación, una vez aprobadas ya son superadas por la realidad y se vuelven obsoletas.

Bajo esta premisa, se analizan las ciudades de Antofagasta y La Serena-Coquimbo, ubicadas en la planicie costera del norte de Chile, buscando identificar patrones urbanos y sus impactos en los mercados de vivienda y suelo. Antofagasta (380 695 habitantes, Censo 2017) y La Serena-Coquimbo (412 586 habitantes, Censo 2017) son ciudades que reciben en mayor medida los beneficios de la explotación minera y por ende durante el súper ciclo del cobre, (desde 2003 hasta 2016), ambas ciudades crecieron en habitantes y edificaciones de forma inédita. En ese sentido su complejidad, como sus ingresos promedio se observaron al alza. Además, ambas comunas, son capitales de su región, son costeras, lineales y se encuentran constreñidas pos la cordillera de la costa hacia el este.

Se logra identificar un subcentro incipiente en Antofagasta y dos aglomeraciones en La Serena, siendo estos de carácter industrial y/o de servicios, sin embargo, al analizar un análisis más claro, pero no es posible determinar sus impactos en los precios, ni en la actividad edificatoria. En ambas ciudades, se identifican aglomeraciones de trabajadores, pero están lejos de vertebrar el territorio, o de aportar a una competencia de otros tipos de viajes con el centro.

\section{Abstract}

This work seeks to identify labor and service sub-centers in the area, using information from the surveys of origin destination of the Chilean Ministry of Transportation, and to analyze the building activity and housing market in each of them. The identification methodology will be followed, established in Marmolejo and Aguirre (2011) regarding the density peaks and the use of worker density, its version of composite density and validating by means of an appropriate version of time density.

There is still a clear relationship between the agglomeration of uses and segregation in the city and the low regulations in the land and housing market. In Chile, there is a low regulation of the housing markets and in particular both cities have regulations at the municipal level, which, given their complexity of approval, once they were approved they are already surpassed by reality and become obsolete.

Under this premise, the cities of Antofagasta and La Serena-Coquimbo, located on the coastal plain of northern Chile, are analyzed, seeking to identify urban patterns and their impacts on the housing and land markets. Antofagasta (380 695 inhabitants, Census 2017) and La Serena-Coquimbo (412 586 inhabitants, Census 2017) are cities that receive the greatest benefits from mining and therefore during the copper super cycle, (from 2003 to 2016), both cities grew in inhabitants and buildings in an unprecedented way. In this sense, its complexity, as well as its average income, were

\footnotetext{
${ }^{1}$ Centro Producción del Espacio Facultad de Arquitectura; diseño y Construcción, Universidad de las Américas Chile, https://orcid.org/0000-0001-7556-8352; ${ }^{2}$ Centro de Política de suelo y valoraciones, Escuela Técnica Superior de Arquitectura de Barcelona, Universidad Politécnica de Cataluña, https://orcid.org/0000-0001-7556-8352; ${ }^{3}$ Centro Producción del Espacio Facultad de Arquitectura; Diseño y Construcción, Universidad de las Américas, Chile, https://orcid.org/0000-0002-1930-4691. * Correo de contacto: caguirre@udla.cl
} 


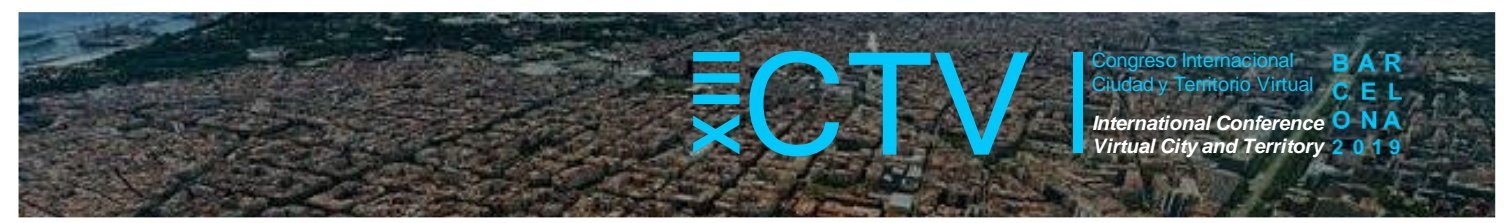

observed on the rise. In addition, both communes are capitals of their region, they are coastal, linear and are constrained by the coastal range to the east.

It is possible to identify an incipient subcenter in Antofagasta and two agglomerations in La Serena, these being of an industrial and / or services nature, however, when analyzing a clearer analysis, but it is not possible to determine their impact on prices, nor in building activity. In both cities, agglomerations of workers are identified, but they are far from structuring the territory, or contributing to a competition of other types of travel with the center.

Palabras Clave: identificación de subcentros, ciudades costeras, baja regulación

Key words: identification of sub-centers, coastal cities, low regulation

\section{Introducción}

El Centro Producción del Espacio ha sido fundado para desarrollar exploraciones críticas de ciudades chilenas utilizando metodologías avanzadas para que las conclusiones se ajusten a las posibilidades de generar soluciones efectivas desde medidas urbanísticas. En este caso, se explora la estructura urbana de Antofagasta, elaborando una descripción de la existencia de potenciales policentros, junto con revisar la accesibilidad socio-espacial a la ciudad desde la perspectiva del ingreso. El objetivo es proveer de un conjunto de resultados que permitan mejorar el diagnóstico de la ciudad de cara al nuevo Plan Regulador Comunal.

Se realiza una aplicación de la teoría de William Alonso indica que existe un vínculo directo en el centro urbano y el valor del suelo, indicando diferentes patrones espaciales que pueden ser aplicables a diferentes ciudades del mundo. La teoría de Alonso de 1964 será basal para el desarrollo de una econometría de las ciudades. En particular, se trabaja sobre la revisión de como el policentrismo impacta sobre la distribución espacial de los proyectos inmobiliarios, en una metodología explorada por Carlos Aguirre y Carlos Marmolejo en 2011, para la ciudad de Barcelona. Para que el análisis sea preciso, se necesita conocer los flujos de personas en la ciudad, dado que la movilidad es fundamental para determinar las centralidades. En este caso, se aplica esta exploración a la ciudad de Antofagasta. Sin embargo, para La Serena Coquimbo, las zonas de transporte son sufrientemente grandes y heterogéneas para permitir un análisis claro de esta estructura, En ese sentido, se aplicará una metodología distinta, con miras a validar el ensayo de análisis para estas ciudades costeras. Se entenderá por subcentro un espacio urbano que cumpla con tres características fundamentales: (i) ser una zona de densidad de empleo significativamente mayor en relación a las zonas vecinas, (ii) donde la densidad relativa es alta y (iii) que represente una intersección con elementos vertebradores de un sistema urbano dentro de una ciudad (Roca, Marmolejo y Moix, 2007).

En esa lógica, cada ciudad debe contener múltiples núcleos (Richardson, 1973), tiene como idea básica, que las ciudades se conforman alrededor de diferentes núcleos, que interactuar entre si. Estos tienen su origen en la integración de centros lejanos en una urbanización rápida al centro urbano, por ejemplo, o como nuevos centros que llenan en vacío entre dos ciudades. Estos núcleos en palabras de Richardson; reflejan cuatro factores principales; la interdependencia, entre algunas actividades; la tendencia que presentan algunas actividades a ser complementarias o afines, como por ejemplo el comercio al por menor y las zonas de oficinas; un antagonismo locacional o exclusión de usos, por ejemplo, industria y vivienda de lujo, y el alto costo del suelo. En ese sentido, Roca (Roca Cladera, 1986), establece que la segregación espacial o socio espacial, es una consecuencia de la competencia por los mejores espacios urbanos. En este punto Alonso, plantea en el apartado de la forma de las ciudades, en que este proceso de competencia a una escala mayor al de la ciudad, puede generar núcleos de precios altos y por ende concentración de determinados usos de suelo. (figura 1) 


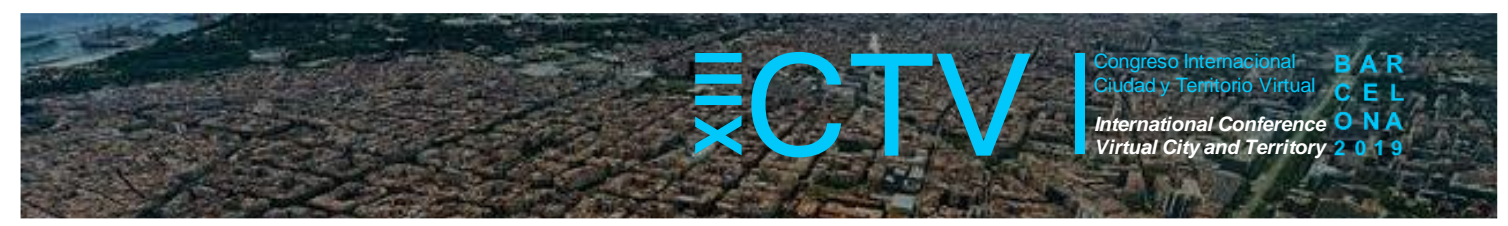

Figura 1. Renta del suelo y patrones de localización a escala Inter ciudades

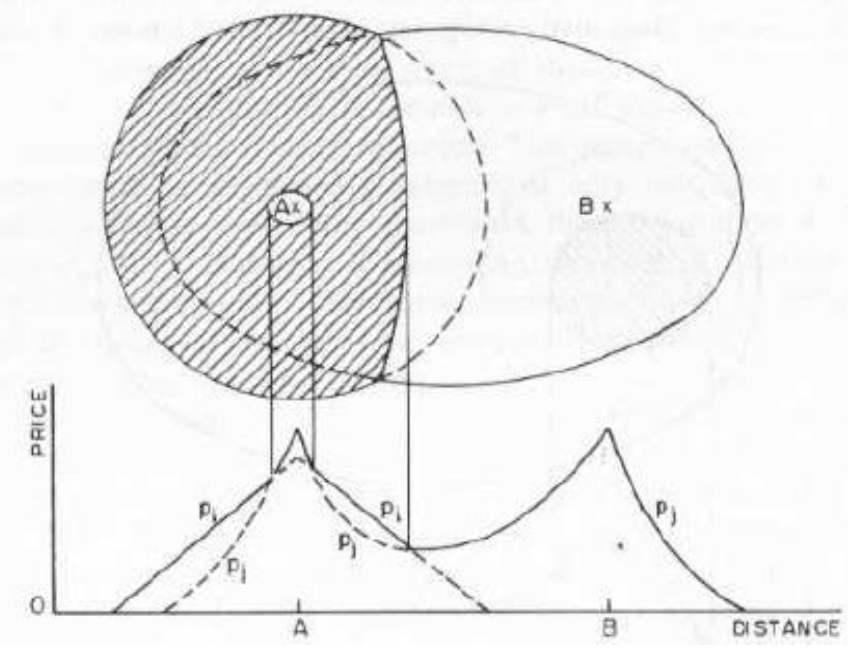

Fuente: (Alonso, 1964)

Esta misma estructura (Shearmur, 2018), revisitando el modelo de centralidad y bid rent de Alonso, casi 50 años después de su primera edición, identifica el aporte de los presupuestos de este último, en especial por la simpleza de la relación entre los lugares de trabajo y los de actividad económica en sus propias palabras "la actividad económica (y su creación de valor concomitante) tiene una ubicación. La actividad económica puede conceptualizarse de varias maneras: generalmente se entiende, al menos cuando se discute la estructura urbana, como una referencia a los establecimientos económicos y a los trabajadores vinculados a estos establecimientos." En ese sentido, un paradigma desarrollado ampliamente en la literatura, de forma empírica y teórica, ha sido la identificación de un CDB y subcentros, (figura 3) cada uno de los cuales interactúa con su zona de influencia local. Hay ejes, tanto radiales como concéntricos, a lo largo de los cuales también se organiza la actividad económica. Algunos de estos ejes estructuran corredores de actividad económica. (Shearmur, 2018)

Figura 2. CBD y subcentralidades, como modelo conceptual

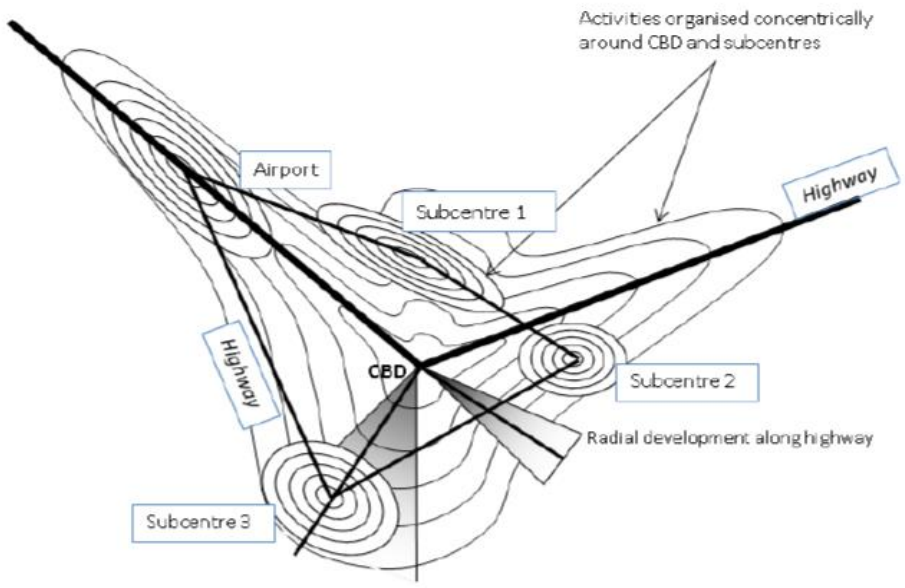

Fuente: Shearmur, 2018

2 Traducción propia 


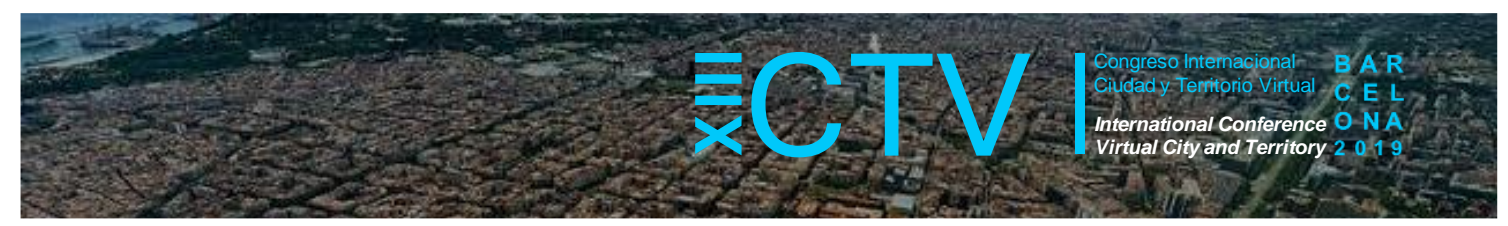

La realidad urbana se encuentra siempre en un margen multidisciplinario, en ese sentido, los acercamientos nacen desde varias vertientes. Intentando dar una explicación para este proceso, Lefebvre (Lefebvre, 1991) plantea que el espacio urbano, se genera en base "tríada conceptual", que se resumen en las practicas, lo concebido y lo representado. (Torres, 2016)

1. Prácticas espaciales (el espacio percibido): abarca la producción y la reproducción, los lugares concretos y las características de los conjuntos espaciales de cada formación social. Aseguran la continuidad y cierto grado de cohesión. En términos de espacio social, y de cada miembro de la relación de una sociedad dada a ese espacio, esta cohesión implica un nivel garantizado de competencia y un nivel específico de rendimiento.

2. Representaciones del espacio (el espacio concebido): están vinculadas a las relaciones de producción y al "orden" que imponen esas relaciones y, por lo tanto, al conocimiento, a los signos, a los códigos y a las relaciones "frontales". "Espacio conceptualizado, el espacio de los científicos, de los planificadores, urbanistas, técnicos e ingenieros sociales (...) quienes identifican lo que es vivido y percibido con lo que es concebido" (Lefebvre, 1991:38). Se trata del espacio dominante en cualquier sociedad (o modo de producción) y es fundamental su influencia en el proceso de producción del espacio y en la actividad productiva de la sociedad.

3. Espacios de representación (el espacio vivido): incorporan simbolismos complejos, a veces codificados, a veces no, vinculados al costado clandestino o marginal de la vida social, como también al arte. Los espacios de representación producen, generalmente, resultados simbólicos.

Si adoptamos esta triada como base epistemológica, podemos hipotetizar cual o cuales de los elementos están involucrados en la discusión de la estructura urbana y del policentrismo. Por ejemplo, las practicas espaciales, se enraízan en la conformación de un barrio, guardan relación con las condiciones espaciales de movilidad cotidiana (vivienda -trabajo, vivienda -comercio 0 vivienda esparcimiento). Por otro lado, la representación, es el esfuerzo de modelar, explicar y producir un espacio desde la teoría o desde la sistematización de datos empíricos. Y por último la representación, que es lo simbólico que está detrás de la percepción y representación del espacio urbano, esta representación, logra plasmar en un símbolo, obra de arte y otro medio, fenómenos sociales complejos y que las dos líneas anteriores no son capaces de captar, ni mesurar de forma eficiente. El mismo Lefebvre (1991), plantea que la ciudad no es ninguno de estos elementos por separado, sino una visión integrada de los elementos de la triada con la complejidad disciplinar de integrarlos. Por tanto, que el acercamiento epistemológico a la estructura urbana policéntrica o policentrismo, es una discusión aun no zanjada y que al menos responde a 4 líneas identificadas por Boix \& Trullen (2012); El policentrismo morfológico, el dinámico, el que se basa en el equilibrio o no de un sistema y, por último, el que le entrega otros nombres a la misma realidad dependiendo de la escala de análisis a la cual se haga referencia. Aun así, esta estructura logra formar un corpus teórico que permite definirla como un programa de investigación.

De Ureña (de Ureña, Pillet, \& Marmolejo, 2013), por su lado, establece una taxonomía, generando dos grandes cercamientos metodológicos, los teóricos y empíricos, segmentando estos últimos en líneas de investigación, en base al propósito y criterios que utilizan, generando además una clasificación geográfica acorde a la naturaleza del territorio en el cual han desarrollado sus marcos empíricos. Según de Ureña (de Ureña, Pillet, \& Marmolejo, 2013), los teóricos se basan en la existencia de un comportamiento racional y económico de los actores y mediante métodos numéricos y algebraicos prueban la existencia de realidades policéntricas. En contrapunto se encuentran los empíricos, quienes buscan establecer mediante el análisis 


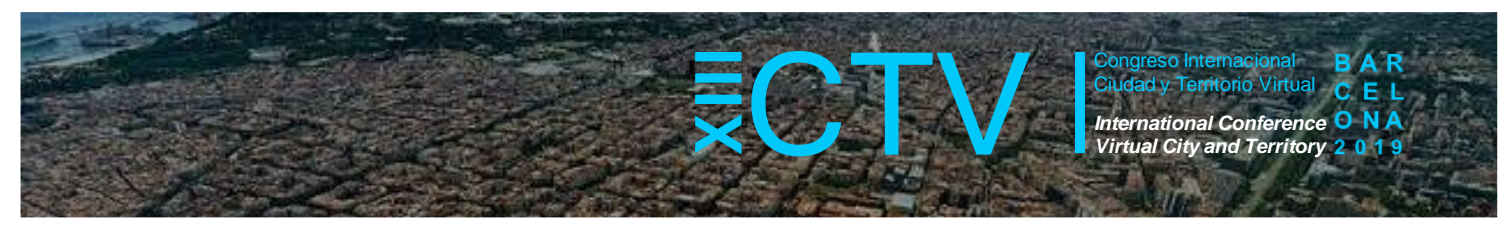

económico y geográfico, las estructuras de las ciudades y los efectos que tiene el policentrismo en ellas. Volviendo los referentes epistemológicos, la complejidad de la ciudad, hace imposible establecer cuál de las familias definida logra abarcar y parametrizar de mejor manera la estructura urbana en ciudades policéntricas. En ese sentido, se pretende establecer una comparación de estos buscando sus convergencias y divergencias asociadas principalmente a las ideas fuerza que subyacen en ellas. Aun así, en la Tabla 1 se muestran las principales familias y su artículo seminal.

Tabla 1. Asociación de los modelos propuestos a sus referentes teóricos

\begin{tabular}{|c|c|c|c|}
\hline Metodología & Trabajo & Variable & Criterio \\
\hline \multirow[t]{14}{*}{ A priori } & Greene (1980) & \multirow{2}{*}{ Medidas oficiales } & \multirow{2}{*}{$\begin{array}{l}\text { Subcentros propuestos por } \\
\text { alguna agencia oficial }\end{array}$} \\
\hline & Griffith (1981) & & \\
\hline & Erickson (1986) & \multirow{2}{*}{ Población } & \multirow{2}{*}{ Tamaño mínimo de población } \\
\hline & Martori y Suriñach (2002) & & \\
\hline & Baerward (1982) & \multirow{3}{*}{$\begin{array}{l}\text { Ubicación de Lugares de } \\
\text { transporte }\end{array}$} & \multirow{3}{*}{ Polos en nodos de transporte } \\
\hline & Erickson y Gentry (1985) & & \\
\hline & Muñiz et al (2003) & & \\
\hline & Dunphy (1982) & \multirow{3}{*}{$\begin{array}{l}\text { Densidad de empleo, } \\
\text { - población factores urbanos, } \\
\text { etc. }\end{array}$} & \multirow{3}{*}{$\begin{array}{l}\text { Estudios de casos con batería } \\
\text { indicadores }\end{array}$} \\
\hline & Scott (1988) & & \\
\hline & Cervero (1989) & & \\
\hline & Bender y Kwang (1985) & \multirow{4}{*}{$\begin{array}{l}\text { Densidad de empleo } \\
\text { Densidad de Población }\end{array}$} & \multirow{4}{*}{$\begin{array}{l}\text { Propuesta de candidatos a priori } \\
\text { y validación mediante } \\
\text { significancia estadística de } \\
\text { función de densidad de empleo o } \\
\text { población }\end{array}$} \\
\hline & Heikkila et al (1989) & & \\
\hline & Dowall y Treffeisen (1991) & & \\
\hline & Shukla y Waddel (1991) & & \\
\hline \multirow{4}{*}{$\begin{array}{l}\text { Flujos de } \\
\text { movilidad }\end{array}$} & Bourne (1989) & Flujos de commuting & Inspección visual \\
\hline & Burns et al (2001) & $\begin{array}{l}\text { Saldo viajes residencia- } \\
\text { trabajo }\end{array}$ & Saldo neto positivo \\
\hline & $\begin{array}{l}\text { Clark y Kuijpers-Linde } \\
\text { (1994) }\end{array}$ & \multirow{2}{*}{$\begin{array}{l}\text { Densidad de generación de } \\
\text { viajes }\end{array}$} & \multirow{2}{*}{$\begin{array}{l}\text { Áreas con una densidad de } \\
\text { generación de viajes }>0.8 \\
\text { desviación estándar }\end{array}$} \\
\hline & Gordon y Richardson (1996) & & \\
\hline \multirow[t]{16}{*}{ Umbrales } & \multirow{2}{*}{ Gulianno y Small(1991) } & \multirow{2}{*}{ Densidad de empleos } & Dempleo>25 empleos/hec \\
\hline & & & Empleos $>10.000$ \\
\hline & \multirow{2}{*}{ Song (1994) } & \multirow{2}{*}{ Densidad de empleos } & Dempleo>37 empleos/hec \\
\hline & & & Empleos $>35.000$ \\
\hline & \multirow{2}{*}{ Cerveró y Wu(1997) } & \multirow{2}{*}{ Densidad de empleos } & Dempleo>17 empleos/hec \\
\hline & & & Empleos >10.000 \\
\hline & \multirow{3}{*}{$\begin{array}{l}\text { Mcmillen y Mcdonals } \\
(1997)(1998)\end{array}$} & \multirow{3}{*}{ Densidad de empleos } & D empleo>25 empleos/hec \\
\hline & & & Empleos $>10.000$ \\
\hline & & & $\begin{array}{l}\text { Gradiente desde subcentro, } \\
\text { negativa y significativa }\end{array}$ \\
\hline & \multirow{2}{*}{ Bogart y Ferry(1999)) } & \multirow{2}{*}{ Densidad de empleos } & Dempleo>20 empleos/hec \\
\hline & & & Empleos >10.000 \\
\hline & \multirow{2}{*}{ Andreson y Bogart(2001) } & \multirow{2}{*}{ Densidad de empleos } & Dempleo>20 empleos/hec \\
\hline & & & Empleos $>10.000$ \\
\hline & \multirow{2}{*}{ Shearmur y Coffey (2002) } & Densidad de empleos & Empleo > 5000 \\
\hline & & Densidad de Población & Empleo/poblacion > 1 \\
\hline & McMillen y Lester (2003) & Densidad de empleos & $\mathrm{De}=15$ empleos/acre $\mathrm{L}=10000$ \\
\hline
\end{tabular}

Fuente: Elaboración propia en base a Muñiz, I. "Barcelona: Ciudad Policéntrica", Working Paper, Universidad Autónoma de Barcelona 2003.

De los modelos identificados, se puede establecer tres líneas de críticas a ellos, la estanqueidad, especialización de los métodos y la sobre estimación del paradigma Monocéntrico de Alonso y Muth. El primer punto débil, desde este análisis, para identificar y validar los subcentros, es la estanqueidad de los métodos y su estructuración. Esto se entiende desde el poco dialogo y complementariedad que muestran al estar basados en variables netamente econométricas o las relacionadas con la movilidad. De hecho, es común que los factores de lugares de trabajo localizados estén no diferenciados los trabajadores residentes y los trabajadores que llegan 


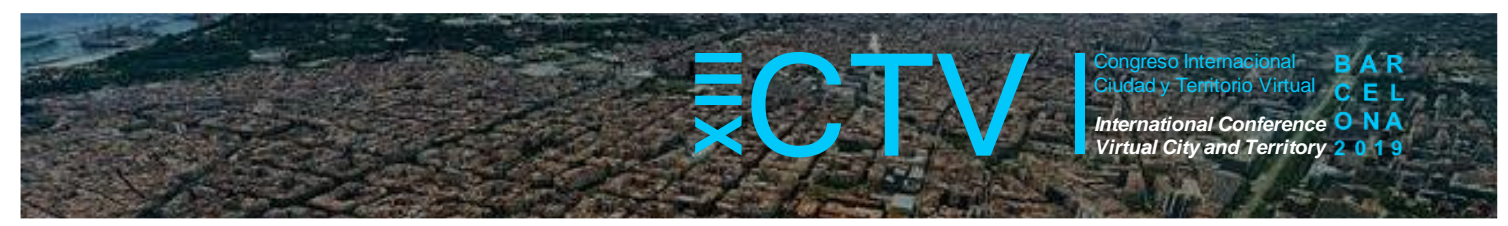

desde otros lugares o salen a trabajar a otros territorios con lo cual la forma de ver el subcentro pierde riqueza. Una Solución a esto sería la generación de medidas de densidad que permitan incorporar estas dimensiones, con lo cual esta pequeña salvedad, podría mejorar las estimaciones de densidad de trabajo, permitiendo la identificación más certera de subcentros, donde los territorios más densos se encuentren definido desde varios ángulos. Si bien es una ventaja que se especialice la técnica, en particular los modelos econométricos, resulta muy difícil su aplicación a varias realidades diferentes. De hecho, McMillen (2003), reconoce de forma certera que el método de Gulianno y Small, es el más fácil de ocupar para comparar ciudades, dado que su agregación permite el desarrollo de puntos anómalos de densidad. En ese sentido, la simpleza y parsimonia de un método de identificación resultaría muy atractiva si se combina con su eficacia.

El aporte de los modelos clásicos de Alonso-Muth, es indudable, es necesario aclarar que sus supuestos básicos, son ampliamente superados por la realidad policéntricas y más aun con las diferentes estrategias de segmentación de mercados inmobiliarios, aun así, que sucede con los supuestos de la centralidad o del monocentrismo, cuando el sistema que se analiza es perfectamente policentrico. Muñiz (2007), plantea la necesidad de incorporar dos aspectos a este análisis, el primero es la delimitación mediante un Filtro urbano, donde las decisiones de ubicación de las familias, es diferente en función de su capacidad económica familiar. El segundo nace de la función de la fiscalidad, o sea como el estado interviene el espacio con subsidios, generando externalidades y a su vez, estableciendo planes de ordenación. Por último, hace patente la necesidad de establecer un modelo ecléctico, en base a las aportaciones de Phe y Wakely, (2000), donde rescata lo mejor de ambos. Uno de sus supuestos que más se hereda en todos los análisis, es la homogeneidad del espacio y por ende la priorización del acceso a los centros de trabajo en las decisiones de residencia (Camagni, 2005). No pocos investigadores, (García López, 2007, Roca, 2007, Muñiz, 2003, Dubin, 1987, Mc Millen, 2003) han criticado este punto y han mejorado sus estimaciones, segmentando la distancia al CBD, en base a ejes de transportes, asociando valores de distancia-tiempo-costo, la geomorfología propia del lugar de análisis, e incorporando las infraestructuras existentes.

Quizás uno de los puntos más complejos es la acción del estado en base a la incorporación de anillos verdes, restricciones a la edificación y edificabilidad, lo que genera niveles importantes de imperfecciones en el mercado de la vivienda y de trabajo ${ }^{3}$. La competencia imperfecta (Santander, 2000) de los mercados inmobiliarios genera este trade off que permite establecer la curva exponencial negativa propia de los modelos monocéntricos. Sin embargo, este trade off es interpretado por el mercado inmobiliario modificando las características de la vivienda, y de mejor manera, modificando los productos ofrecidos en base a la elasticidad precio de los diferentes atributos de la vivienda (Aguirre y León, 2007; Aguirre, 2003, Camagni, 2005). Roca (1982), plantea la realidad de los valores de suelo como segmentada, lo que puede ser extensible a los mercados inmobiliarios, donde cada uno de los submercados inmobiliarios aumentan sus rentas en base a la mejora de sus características.

McMillen y McDonals (2007), establecen un cambio en las funciones que nacen de Alonso-Muth, donde a contar de un punto de distancia del CBD, los valores inmobiliarios y de densidad de población empiezan a crecer (Figura 1). En ese sentido, Muñiz (2003) ha generado modelos de cubic spline para identificar "grumos" de densidad de población, aportando con esta técnica de

\footnotetext{
${ }^{3}$ Los Polígonos industriales, las zonas de protección, etc.
} 


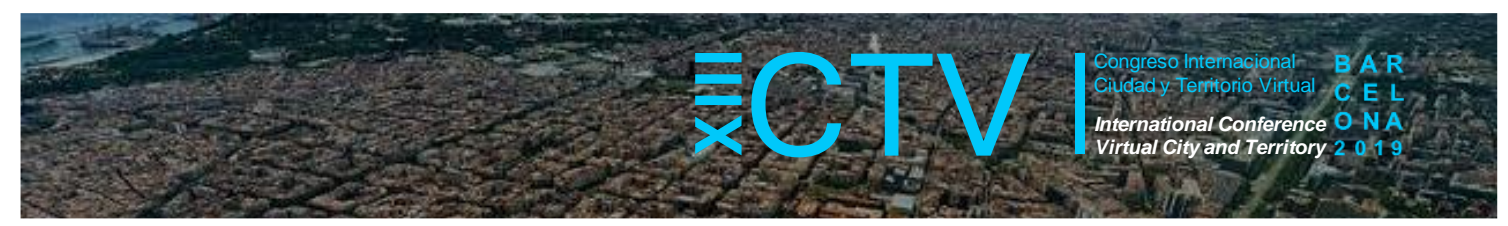

análisis. Sin embargo, resulta compleja su aplicación ya que las escalas de medición deben corresponder a secciones censales o incluso con mayor representatividad espacial. Para diferentes ciudades, se ha probado el análisis de modelos de precios, sin embargo, resulta complejo su análisis, ya que econométricamente, alguno de sus valores tiene a ser no representativos y más aún existe una multicolinealidad efectiva entre las medidas de distancia y accesibilidad de los centros y subcentros.

En ese sentido, y tratando de explicar estos cambios de pendiente, Muñiz (2007) citando los trabajos de Muth (1969), Mills (1973), Johnson y Kau (1980), Alperrovich (1983), incorpora una formulación donde fuera de la distancia se incorporan otros factores asociados a la demografía, geografía y socioeconómicos, para mejorar las estimaciones de densidad de población. En esa línea, (Straszeheim,1987), establece que el modelo de Alonso-Muth al estar basado en un modelo de consumo de tierras, ignora efectos como el vecindario o barrio, las características de las condiciones socioeconómicas, la densidad de población, las características ambientales y los servicios públicos y privados que existen en la zona. Por tanto, un modelo econométrico de precios hedónicos debe estar especificado con estos valores tratando de mostrar el vector general de con estas condiciones donde sea posible observar el comportamiento del precio de un bien en particular cuando se produce una variación en alguna de sus características. Asimismo, es muy complejo establecer esto elementos en ciudades intermedias, tanto por la ausencia de datos de calidad espacial y regularidad suficiente como también, la poca precisión espacial de estos. En ese sentido, este trabajo busca explorar dos ensayos metodológicos de identificación de subcentraldiades para dos ciudades costeras de Chile, y desde una perspectiva heterodoxa, permitir una aproximación a la subcentradlidad en dos tipos de ciudades chilenas.

\section{Antofagasta y La Serena-Coquimbo, ciudades costeras}

Antofagasta y la Serena son dos ciudades del norte de Chile, en ambos casos se dan morfologías lineales y que se plantean como ciudades de vacaciones y de trabajo minero, En esa lógica, presentan algunas diferencias, por ejemplo, mientras que La serena es una de las ciudades con mejor calidad de vida, mientras que Antofagasta es todo lo contrario. Aun así, Antofagasta es la ciudad con el PIB per cápita más alto de Chile.

En el caso de Antofagasta, se constata existencia de una ciudad con un fuerte patrón lineal encofrado entre el rocoso borde costero marino y el pie de monte de la cordillera de la Costa, un aspecto particular entre las capitales regionales de Chile, dado que esta distancia es bastante escueta, lo que produce condiciones geográficas que empujan la expansión urbana hacia sus extremos norte y sur. Un segundo aspecto relevante para considerar es la existencia de segregación socio-residencial, como ocurre en diferentes ciudades de Chile. Esta segregación podría tener impacto en la configuración de subcentros, en cuanto estos podrían fomentar la concentración en sectores donde el valor de suelo es más alto. Para testear estas condiciones, la linealidad y la segregación, en referencia a sus impactos sobre la estructura urbana de la ciudad, se utilizan los datos de la Encuesta Origen Destino del año 2010 y los precios del suelo y la vivienda entre 2012 y 2019. Con estos factores se construye una hipótesis de configuración urbana.

En el caso de La Serena, denominada como conurbación de La Serena- Coquimbo constituye un sistema urbano de ciudades intermedias, localizado en el centro norte de la República de Chile. Con 448.784 habitantes (INE, 2017), ha presentado un sostenido aumento de población y 


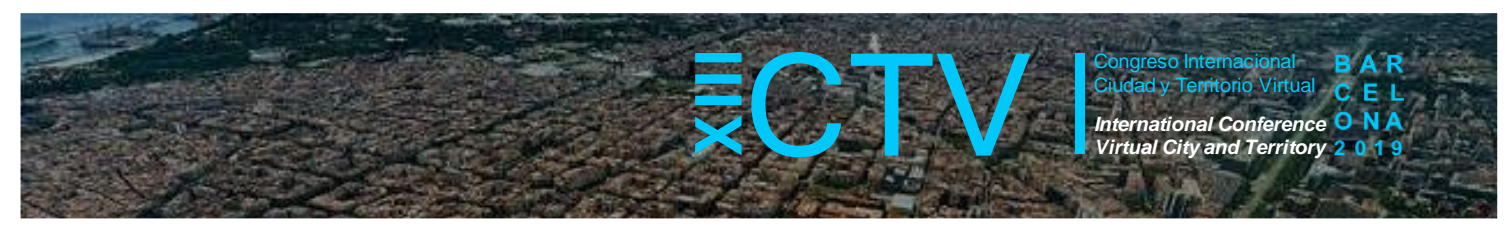

superficie de su mancha urbana en las últimas tres décadas, encaminándose a ser declarada la cuarta área metropolitana del país. Sin embargo, la ciudad enfrenta importantes desafíos en su conformación como sistema metropolitano. La geomorfología de su emplazamiento presenta barreras naturales al desarrollo urbano y una alta vulnerabilidad ante tsunamis; el dinamismo inmobiliario y la fuerte fluctuación de población flotante de carácter estacional contrastan con un sistema vial restringido, acotado geográficamente y en la actualidad ineficiente y una planificación fragmentada. En esa lógica, los modelos datos disponibles de Encuestas origen destino, se refieren a macrozonas que reflejan esta fragmentación. Además, la condición lineal de la conurbación, resulta necesariamente fragmentada y por ende un análisis de densidades de trabajadores, no presenta resultados favorables. En ese sentido, se prefirió desarrollar un análisis de los usos de suelos declarado en el catastro. En ese caso, se desarrolla una selección de los usos de suelos que guardan relación con la generación de trabajo y, asimismo, su concentración, especialización espacial y diversidad.

\section{Definición de los métodos de análisis}

Para empezar, se debe indicar que este ensayo exploratorio, busca probar métodos heterodoxos de identificación de subcentraldiades en ciudades costeras, lineales y con un fuerte componente turístico, En esa lógica, se prueban dos métodos distintos uno asociado a la movilidad entre zonas y se valida de forma descriptiva, y el segundo, se analiza el uso de suelo como variable registral y articulará del espacio.

En el caso de Antofagasta se estableció un análisis de los precios del suelo y de la Encuesta origen destino, el ministerio de transporte s de Chile, Para el caso de la Serena Coquimbo se accedió al catastro de bienes inmuebles del servicio de impuestos internos, analizando los diversos usos y asumiendo algunas condiciones de estos. Además, se coteja con los valores de precio de suelo de la comuna para el mismo año de la última EOD, disponibles en el Observatorio de ciudades UC.

Se realiza un análisis asociado a la regresión exponencial, que permite establecer una bid rent de referencia y posteriormente analizar los residuos entre este valor resultante de la regresión y los valores medidos. (figuras 3, 4 y 5) La bid rent de precios, no manifiesta valores de residuos que permitan establecer algún valor de subcentralidad. Por tanto, podemos indicar que esta ciudad no cuenta con subcentralidades evidentes desde sus valores de suelos. Sin embargo, en una segunda etapa se analizan los resultados de la EOD donde se presentan los valores de densidad de trabajadores.

En este punto de análisis, se observa un viso de subcentralidad en la zona industrial cercana al centro. Esta zona atrae una cantidad importantes de trabajadores, genera viajes a las otras zonas de la ciudad. (figura 6). Al analizar los viajes, tanto porcentualmente como mediante un análisis de componentes principales, se observa dos elementos. El primero es el desarrollo porcentual de las llegadas y salidas a la zona industrial, (figura 7), donde se observa cómo se mueven los trabajadores desde y hacia el distrito, poniendo en cuestión el proceso de atracción de esta zona como subcentralidad.

Además, se observa un análisis ortogonal de las dimensiones subyacentes de los datos de movilidad, Obteniéndose 4 cuadrantes (figura 8), donde se observa un patrón de viajes asociados desde el centro y la zona industrial. 


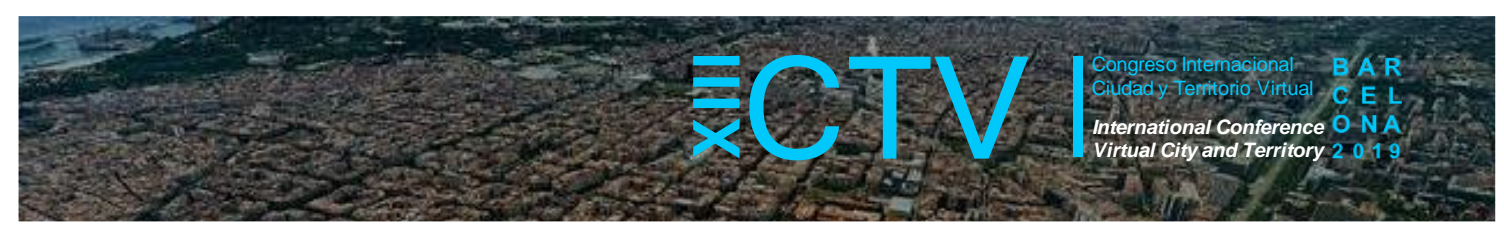

Figura 3. Antofagasta y precios de suelo

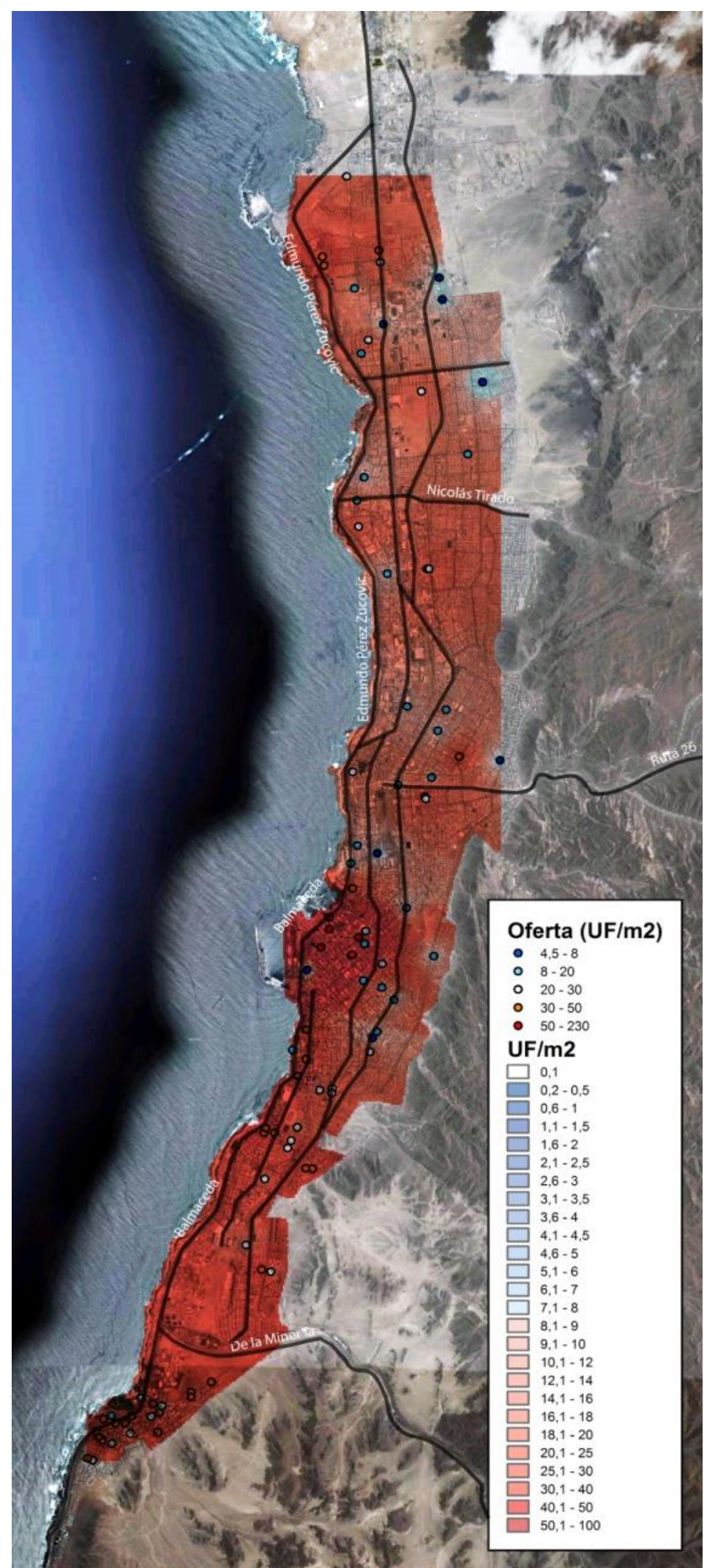

Fuente: Observatorio de Ciudades UC. 
Figura 4. Antofagasta y precios de suelo, con sus áreas asociadas

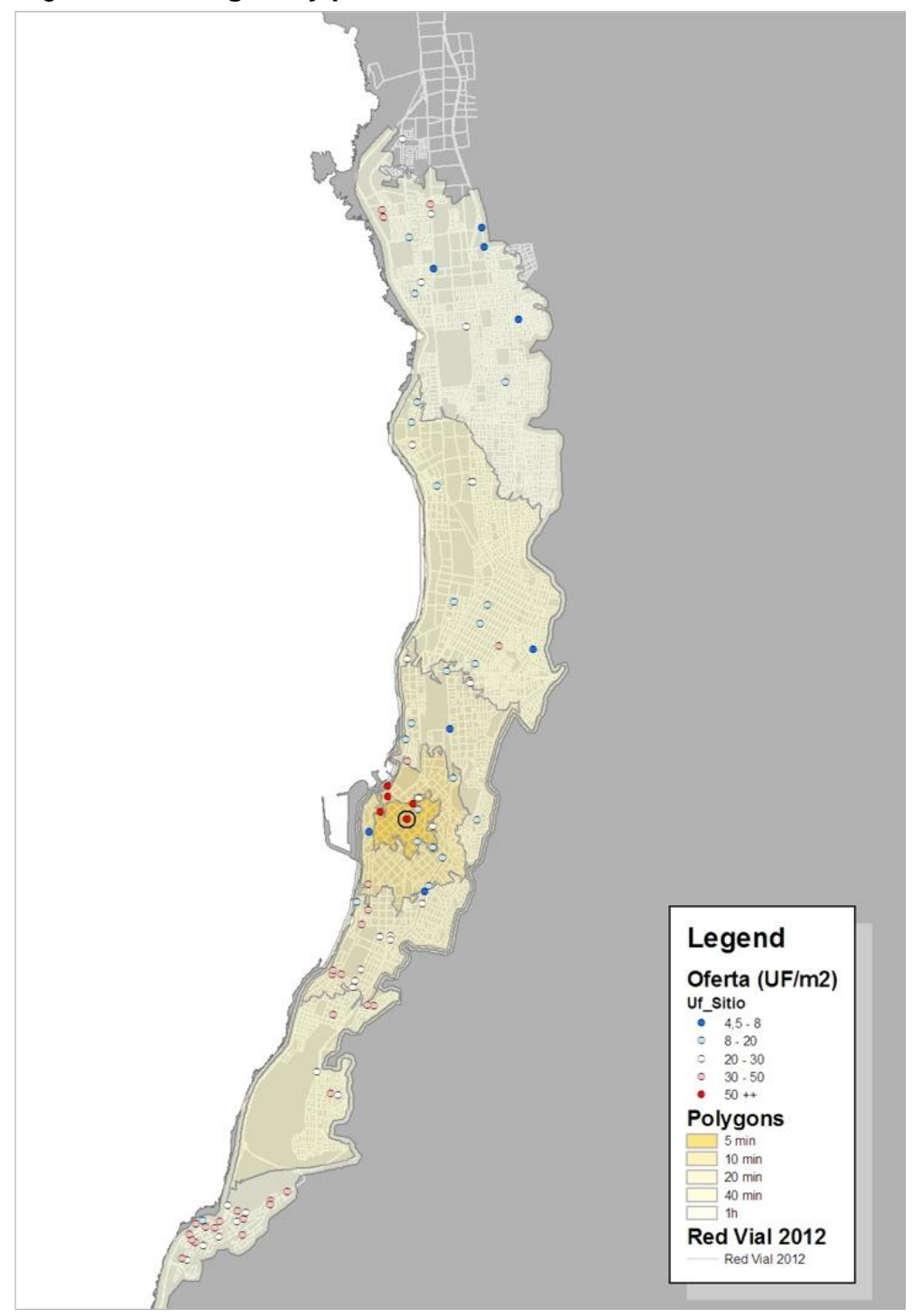

Fuente: Observatorio de Ciudades UC.

Figura 5. Antofagasta y precios de suelo, bid rent

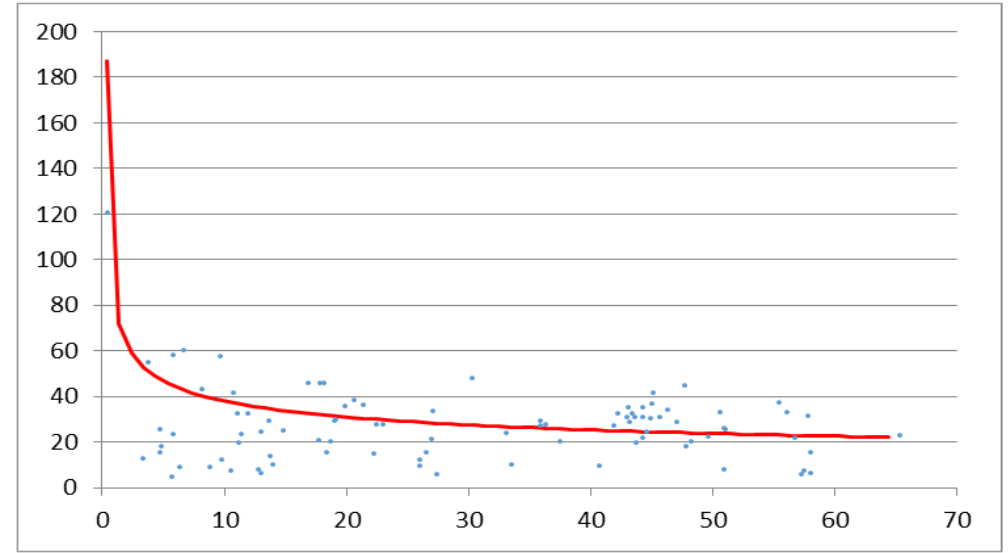

Fuente: Elaboración propia en base a Observatorio de Ciudades UC. 
Figura 6. Antofagasta y densidad de trabajadores

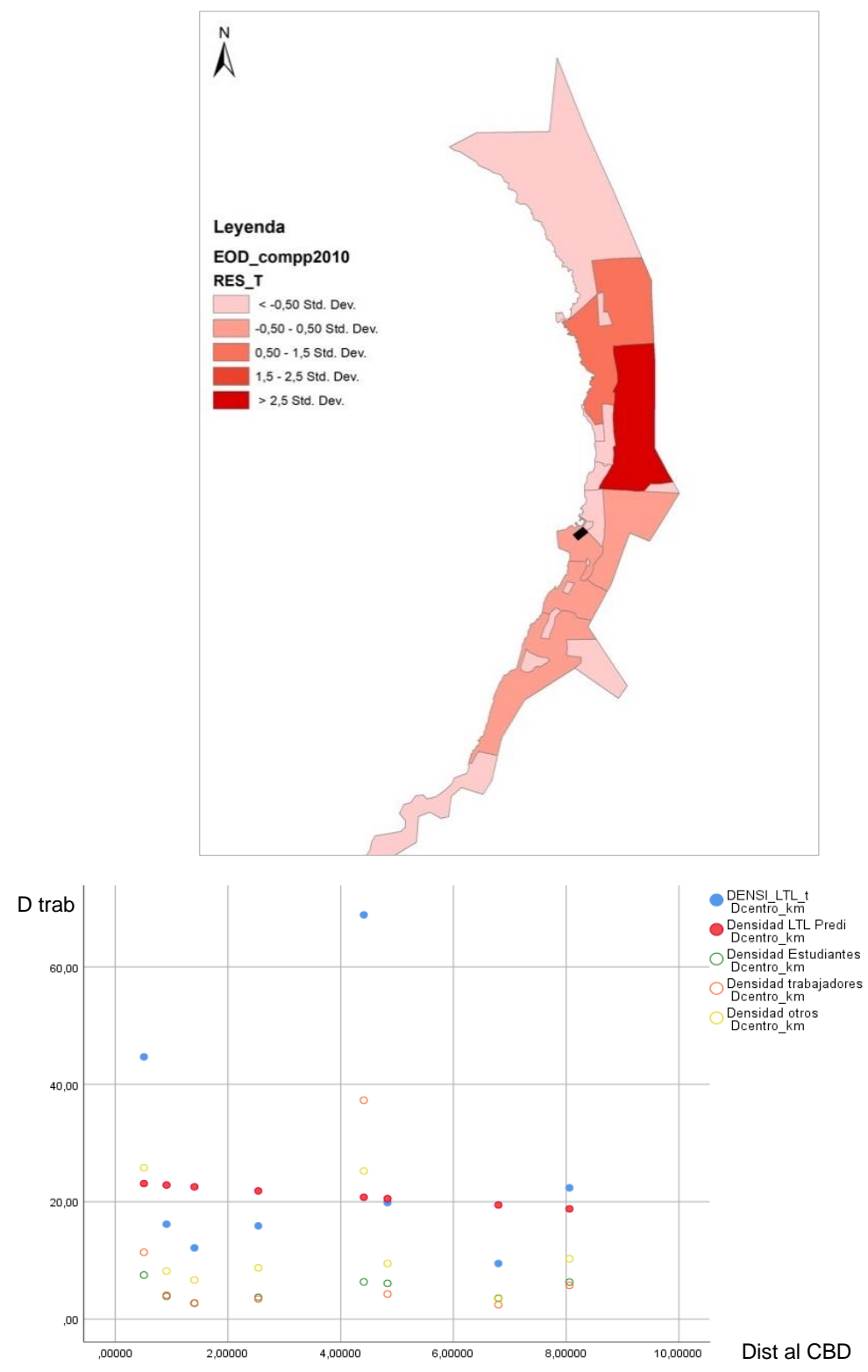

Fuente: Elaboración propia en base a Encuesta Origen Destino.

Figura 7. Viajes con destino trabajo en las distintas zonas de la EOD

\begin{tabular}{|c|c|c|c|}
\hline \multicolumn{2}{|c|}{ Entradas } & \multicolumn{2}{|c|}{ Salidas } \\
\hline 01_Centro & $12 \%$ & 01_Centro & $13 \%$ \\
\hline 02_Centro Sur & $5 \%$ & 02_Centro Sur & $6 \%$ \\
\hline 03_Centro Norte & $7 \%$ & 03_Centro Norte & $6 \%$ \\
\hline 04_Centro Oriente & $7 \%$ & 04_Centro Oriente & $7 \%$ \\
\hline 06_Oriente & $29 \%$ & 06_Oriente & $28 \%$ \\
\hline 07_Norte & $22 \%$ & 07_Norte & $23 \%$ \\
\hline 08_Sur & $4 \%$ & 08_Sur & $3 \%$ \\
\hline
\end{tabular}

Fuente: Elaboración propia en base a Encuesta Origen Destino. 


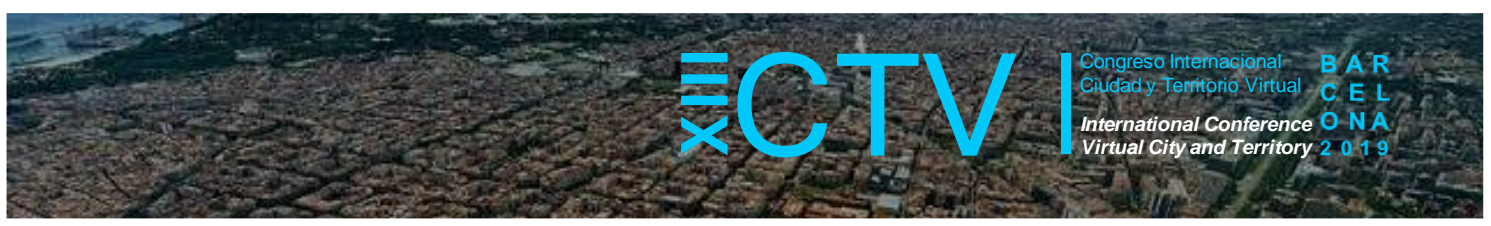

Figura 8. Puntuación de Componentes principales de los viajes con destino trabajo

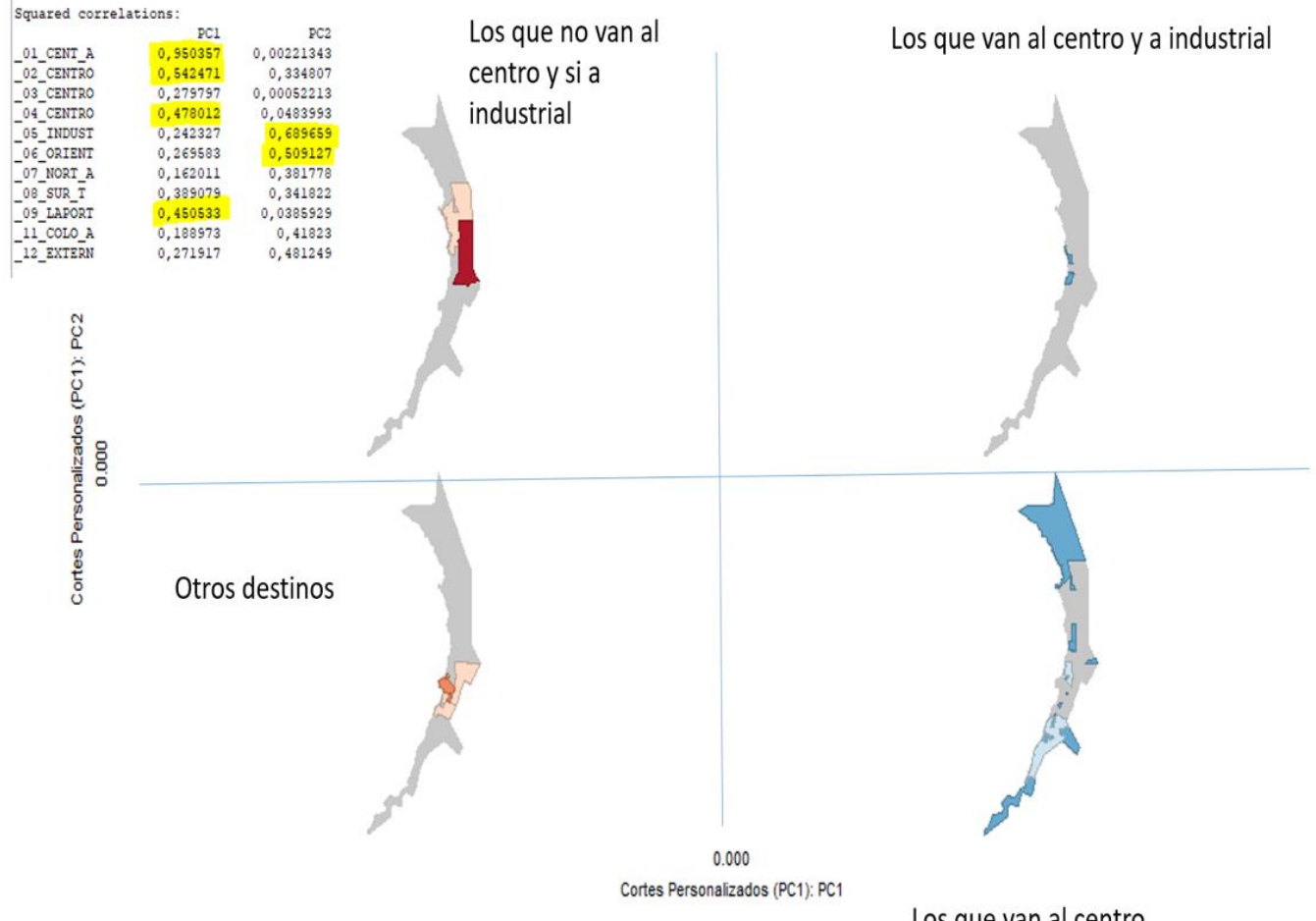

Los que van al centro

Fuente: Elaboración propia en base a Encuesta Origen Destino.

\section{La Serena Coquimbo}

Este es un estudio cuantitativo de carácter inductivo y exploratorio. Metodológicamente se orienta en el índice de especialización territorial. El estudio de especialización territorial permite generar interpretaciones más complejas de la organización urbana (Fritzsche \& Vio, 2000). Lo valioso de esta aproximación es que permite revisar las sub-centralidades que no necesariamente coinciden con el distrito de empleo y negocios de cada ciudad (Chica \& Marmolejo, 2011), algo que en Chile al menos no está disponible en la información oficial por lo que este artículo contribuye a llenar dicho vacío también.

Así, la exploración desarrollada en este artículo permite determinar la especialización de actividades de una ciudad a partir de identificar cómo interactúan diversos giros económicos por manzana. Esto ha sido posible a partir de información del Servicio de Impuestos Internos. Dicho de otra manera, el estudio determina en qué espacios de la ciudad se produce una mayor concentración de actividades económicas diversas y con ello se presume que en dichos espacios se produce una mayor interacción entre personas.

En ese sentido se eligen los usos de suelo, comercial, industrial, educación, culto, recreación y oficinas. A nivel de manzana se obtiene la Tabla 2, donde se observan las manzanas que cumple la condición de máxima especialización en los usos que atraen viajes, como también una escaza diversidad de usos (figura 9), que se observan gráficamente en la figura 10. 
Tabla 2. Estadígrafos de metros cuadrados e indicadores, a escala manzana

\begin{tabular}{|c|c|c|c|c|c|c|}
\hline & $\begin{array}{l}\text { Superficie por } \\
\text { manzana de } \\
\text { destinos } \\
\text { atractores }\end{array}$ & $\begin{array}{l}\text { Superficie por } \\
\text { manzana de } \\
\text { destino } \\
\text { Habitacional }\end{array}$ & $\begin{array}{l}\text { Índice de } \\
\text { especialización } \\
\text { de destinos } \\
\text { atractores }\end{array}$ & $\begin{array}{l}\text { Índice de } \\
\text { especialización } \\
\text { de destino } \\
\text { Habitacional }\end{array}$ & $\begin{array}{l}\text { Índice de } \\
\text { diversidad } \\
\text { de usos } \\
\text { de suelo }\end{array}$ & $\begin{array}{l}\text { Total de } \\
\text { superifices } \\
\text { Manzana }\end{array}$ \\
\hline $\mathrm{n}$ & 3593 & 3593 & 3593 & 3593 & 3593 & 3593 \\
\hline Media & 454,58 & 2707,94 & 0,549 & 1,195 & 0,235 & 3691,537 \\
\hline Mediana & 0 & 1277 & 0 & 1,363 & 0 & 1465 \\
\hline Moda & 0 & 0 & 0 & 1,4 & 0 & 984 \\
\hline Desv. típ. & 3018,474 & 4159,927 & 1,4222 & 0,3369 & 0,389 & 8520,6443 \\
\hline Suma & 1633310 & 9729619 & 1970,9 & 4295,1 & 844,5 & 13263694 \\
\hline \multicolumn{7}{|l|}{ Percentiles } \\
\hline 25 & 0 & 687,5 & 0 & 1,227 & 0 & 833 \\
\hline 50 & 0 & 1277 & 0 & 1,363 & 0 & 1465 \\
\hline 75 & 40,5 & 2796,5 & 0,189 & 1,363 & 0,319 & 3743 \\
\hline
\end{tabular}

Fuente: Elaboración propia en base a datos del Servicio de Impuestos Internos.

Figura 9. Distribución del índice de especialización territorial y de diversidad de usos por manzana
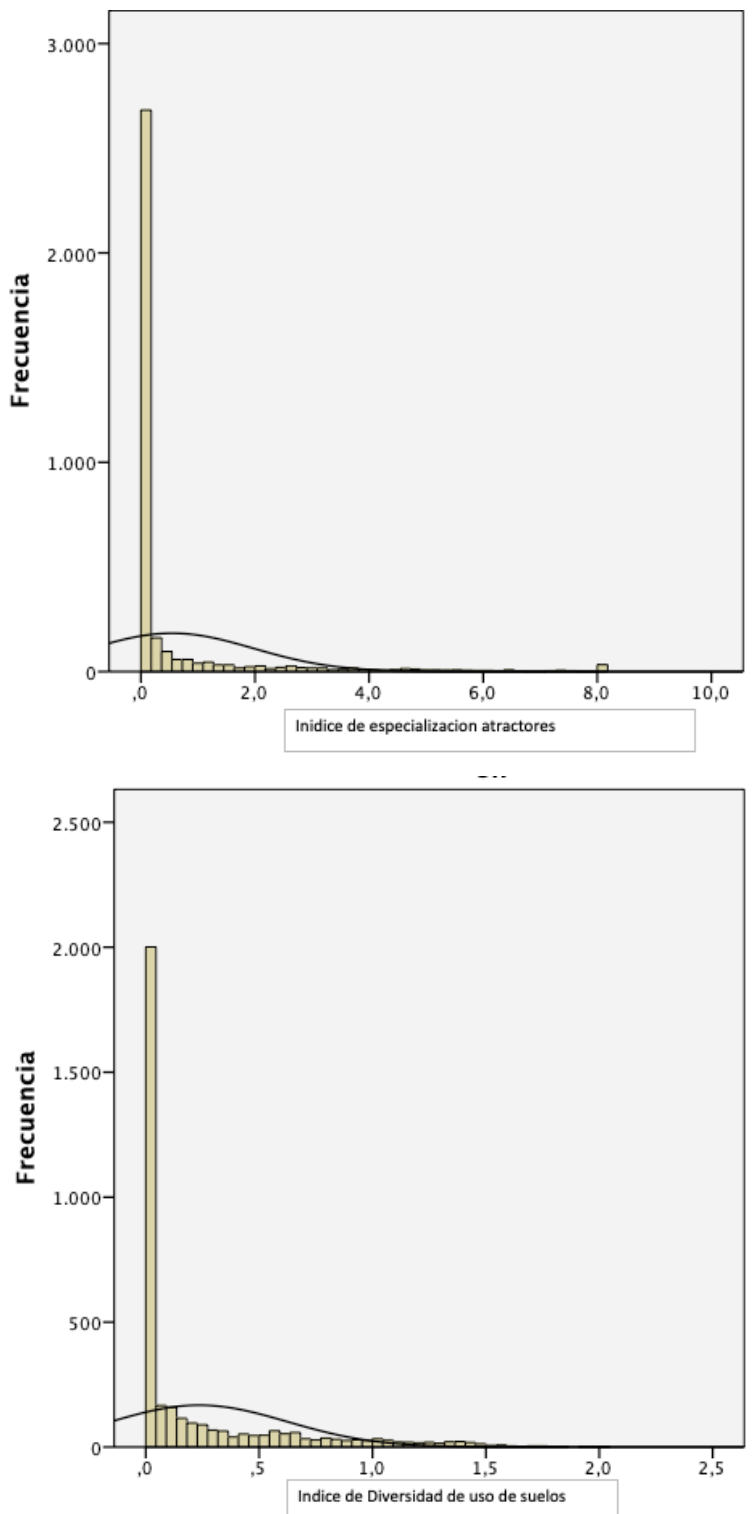

Fuente: Elaboración propia en base a datos del Servicio de Impuestos Internos 
Figura 10. Comparación de índices y cuadrantes de caracterización de las manzanas

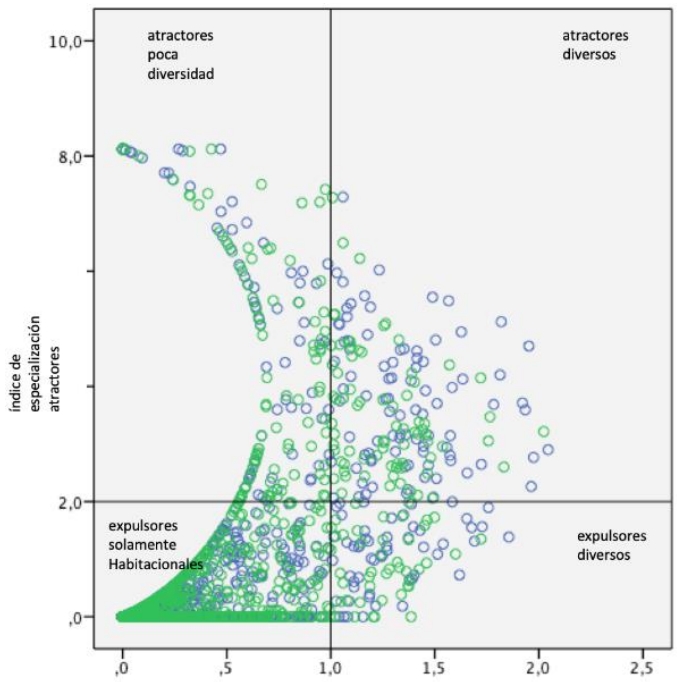

Fuente: Elaboración propia en base a datos del Servicio de Impuestos Internos.

Figura 11. Manzanas resultantes del análisis y candidatas a subcentralidad

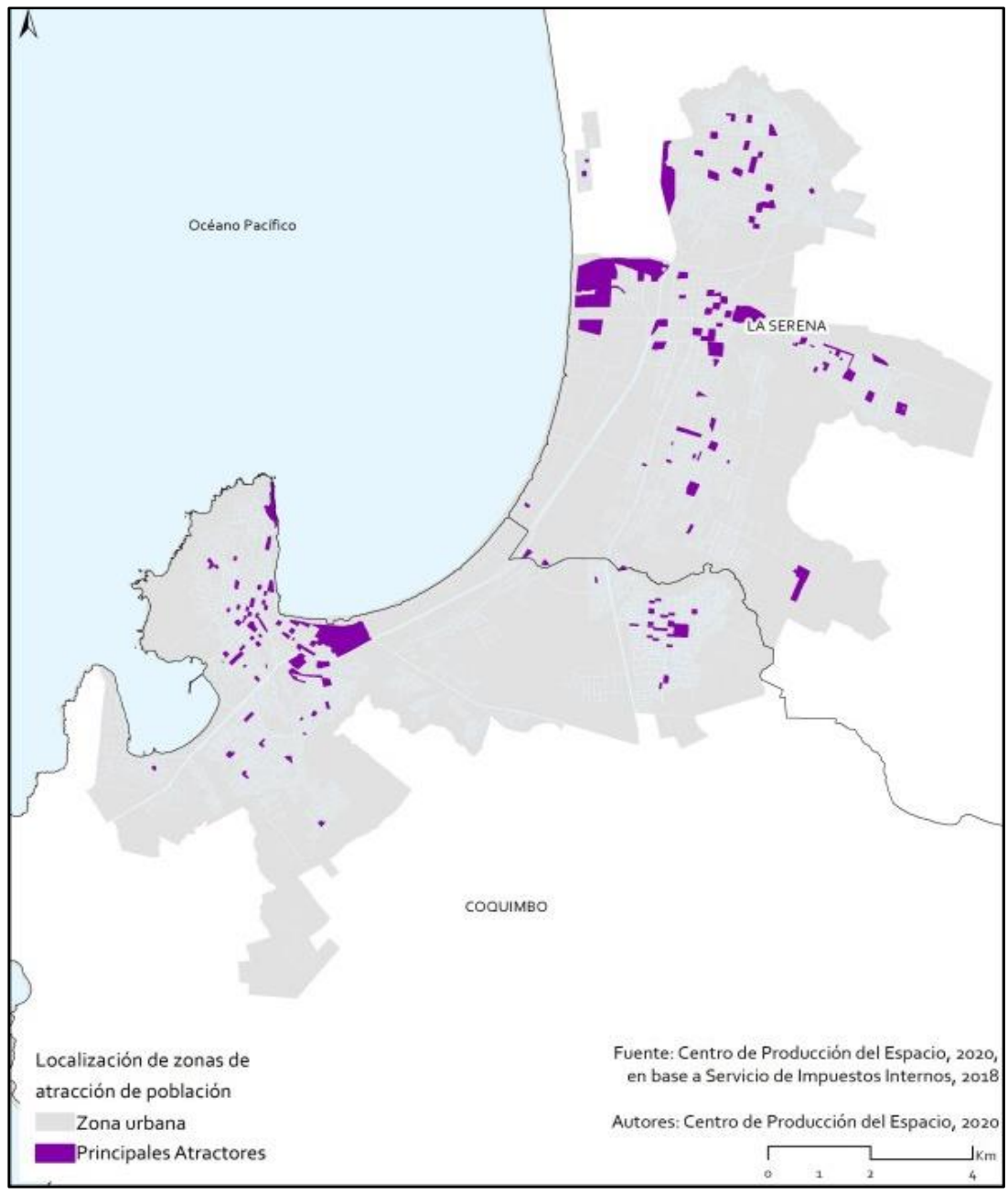

Fuente: Elaboración propia en base a datos del Servicio de Impuestos Internos. 


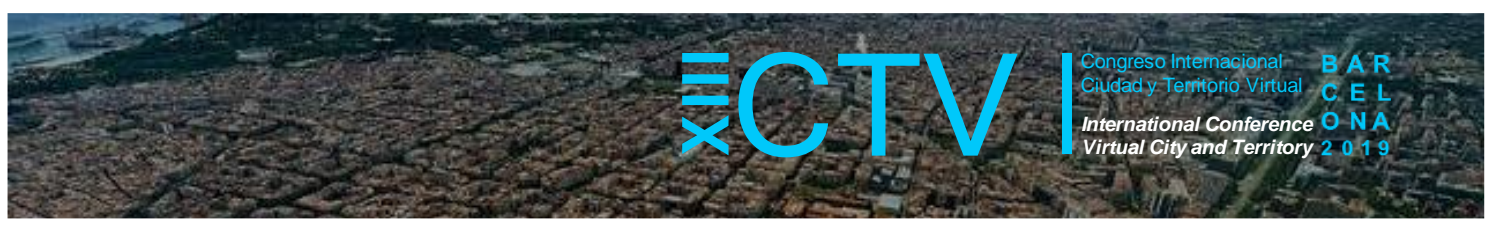

Figura 12. Manzanas atractoras de viajes y manzanas habitacionales

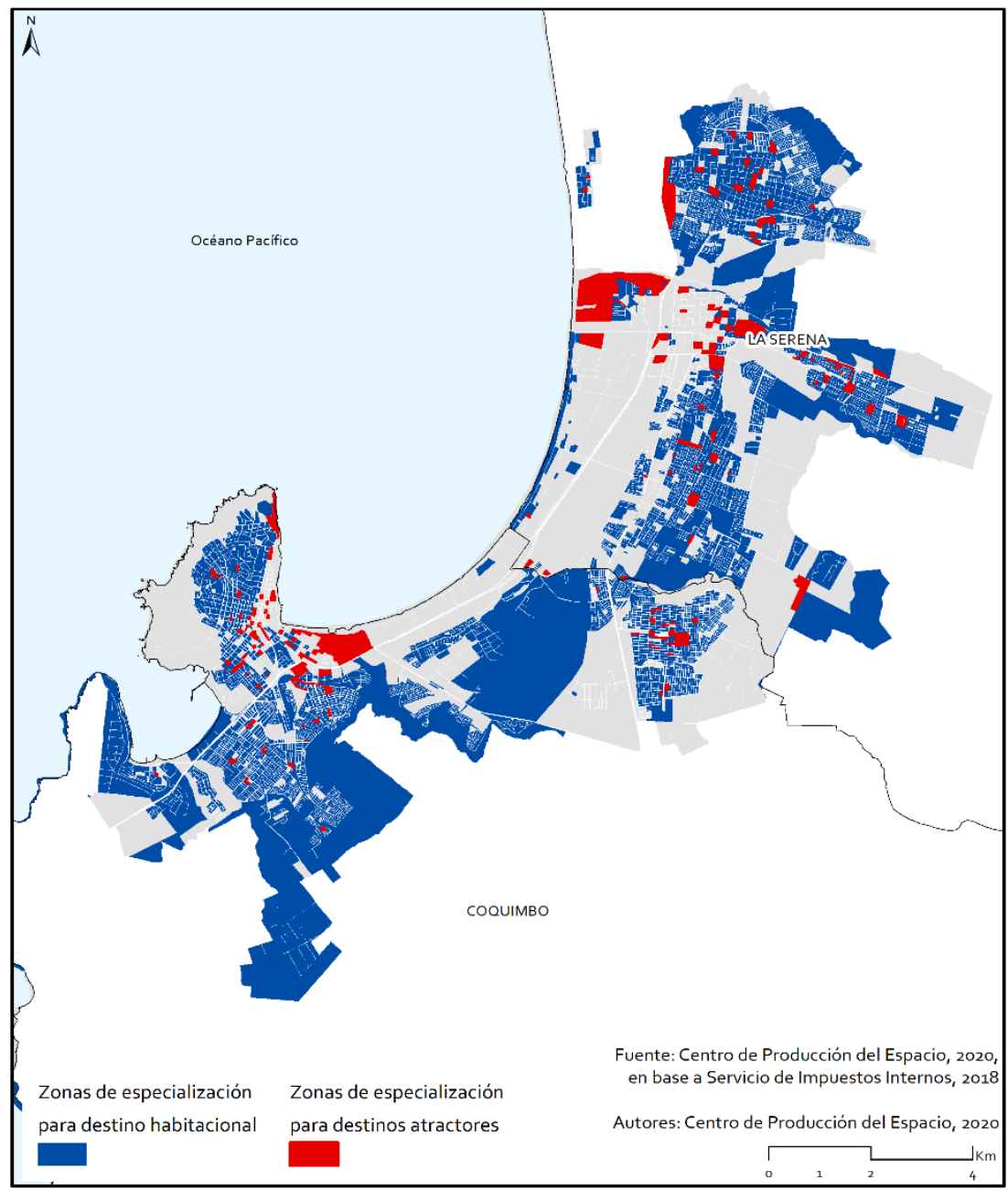

Fuente: Elaboración propia en base a datos del Servicio de Impuestos Internos.

\section{Reflexiones finales}

Esta exploración, expresada en este artículo, busca establecer formas alternativas de identificación de subcentralidades en ciudades intermedias chilenas, con distintos alcances, datos basales y metodologías. Si bien las familias funcional y densidades, han ganado su espacio en la bibliografía, no se han explorado métodos más heterodoxos en términos metodológicos, haciendo cruces entre el la movilidad y la densidad.

Esto último es particularmente relevante en ciudades intermedias cuyo proceso de metropolización esta aun iniciándose. Así, las nuevas visiones "indisciplinadas" o en eclécticas en términos de sus preceptos o líneas de investigación, permitirían ver los fenómenos más incipientes, y subcentralidades más liquidas y difusas. En esa lógica, los modelos acá presentados, uno asociado a la determinación de las condiciones de movilidad mediante una síntesis de viajes entre zonas mediante un análisis de componentes principales y una caracterización de estos, mediante el puntaje factorial de cada zona. Este modelo permite evidenciar la una subcentralidad incipiente en la estructura urbana de Antofagasta, asociada a las medianas empresas industriales. 


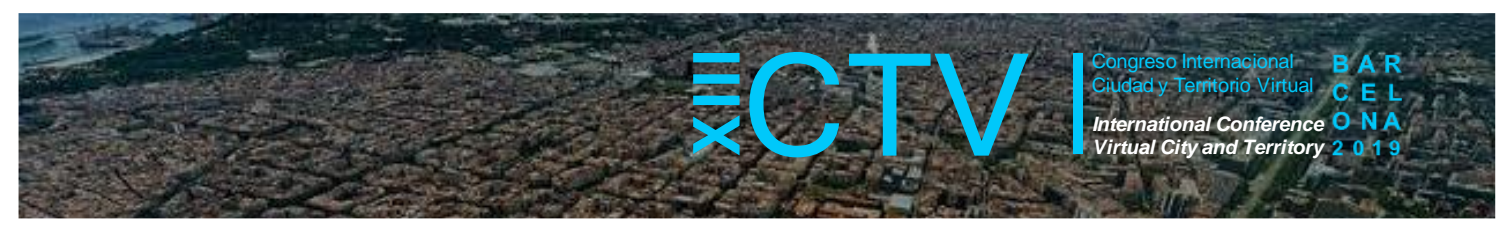

En el caso de una ciudad intermedia con mayor fragmentación, y con una clara conurbación entre dos núcleos urbanos, La Serena y Coquimbo, en análisis de los usos de suelo del catastro de los impuestos, permite identificar las manzanas con mayor atracción de viajes, graficando una versión de subcentralidiades barriales, para cada fragmento y para el sistema de ciudades. Las proyecciones, es probar las metodologías en otras ciudades intermedias, con distintos niveles de fragmentación, y comparar los resultados.

Agradecimientos: Esta investigación fue desarrollada con fondos del CPE UDLA y el apoyo de CPSV de la ETSAB Barcelona. Se agradece al geógrafo Juan Correa, por toda su ayuda con las cartografías, como también a Felipe Morales y Ricardo Trufello del Observatorio de Ciudades PUC, por el acceso a información histórica de precios de suelo en el marco del proyecto MEDIT (2007-2012)

Contribuciones de los autores: Carlos Aguirre desarrollo la metodologías y exploración de datos, Además se encargó de la redacción del artículo, Carlos Marmolejo, desarrollo el análisis crítico de las metodologías y Francisco Vergara, Desarrollo parte de las metodologías de análisis de datos, como también el plan de redacción del artículo.

Conflicto de Intereses: Los autores declaran que no hay conflicto de intereses.

\section{Bibliografía}

Alonso, W. (1964), Location and Land Use, Cambridge, Mass., Harvard University Press.

Aguirre, C., Ramos, R., (2005), Impacto del ruido urbano en el valor de los departamentos nuevos: un estudio de precio hedónico aplicado a bienes ambientales. Revista de la Construcción, volumen 4, número 1, páginas 59-69.

Aguirre, C; Marmolejo C. (2009). "Effects of labor subcentres in urban property values. Case study of the metropolitan region of Barcelona". 49th European Congress of the Regional Science Association International, 29-30 de Agosto, Polonia.

Aguirre, C, \& Marmolejo, C. (2011). El impacto del policentrismo sobre la distribución espacial de los valores inmobiliarios: un análisis para la Región Metropolitana de Barcelona. Revista de la construcción, 10(1), 78-90. DOI https://dx.doi.org/10.4067/S0718-915X2011000100008

Anas, A., \& Kim, I. (1996). General Equilibrium Models of Polycentric Urban Land. JOURNAL OF URBAN ECONOMICS, 232-256.

Bertaud, A. (2002). The Spatial Organization of Cities: Deliberate Outcome or Unforeseen Consequence?" World Development Report, Dynamic Development in a Sustainable World. Background Paper.

Burns, M., Moix, M., Roca, J. (2001). Contrasting Indications of Polycentrism within Spain's Metropolitan Urban Regions, Eighth European Estate Society Conference, Alicante, Junio 26-29.

Roca Cladera, J., \& Moix, M. (2005). The interaction value: its scope and limits as an instrument for delimiting urban systems. Regional Studies, 359-375.

Coombes, M., Openshaws, S. (1982): The use and definition of travel-to-work areas in Great Britain: some comments", Regional Studies, 16, 141-149. 


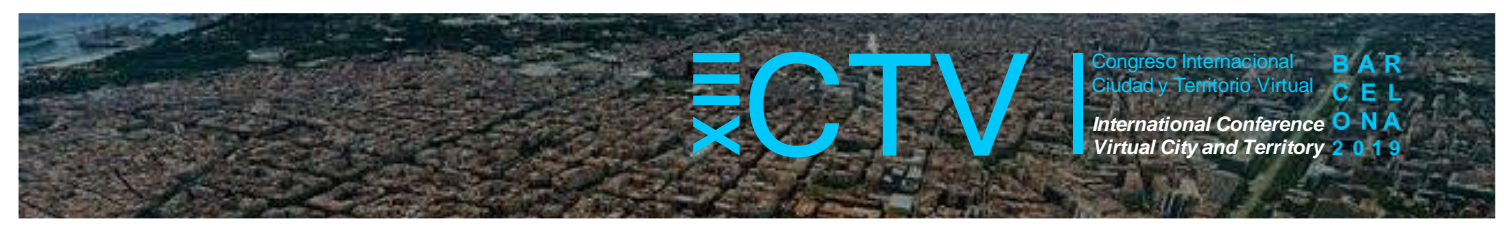

Chica Mejía, E., \& Marmolejo Duarte, C. R. (2011). Los sectores económicos intensivos en conocimiento y sus formas de localización en el territorio metropolitano: un estudio para la Región Metropolitana de Barcelona. ACE: architecture, city and environment, 6(16), 223-252. DOI https://dx.doi.org/10.5821/ace.v6i16.2525

Craig, S.G., NG, P.T. (2001) Using Quantile Smoothing Splines to Identify Employment Subcentres in a Multicentric Urban Area, Journal of Urban Economics, 49, pp. 100-120.

Fujita, M., Krugman, P, Venables, P. (2000). Economía Espacial, Las ciudades, las regiones y comercio internacional", 1era Edición, Ariel Economía, Barcelona.

Fritzsche, F. J., \& Vio, M. (2000). Especialización y diversificación industrial en la Región Metropolitana de Buenos Aires. EURE (Santiago), 26(79), 25-45. Recuperado de https://scielo.conicyt.cl/scielo.php?pid=s0250-71612000007900002\&script=sci arttext

García-López, M.A. (2007). Estructura Espacial del Empleo y Economías de Aglomeración: El Caso de la Industria de la Región Metropolitana de Barcelona, Architecture, City \& Environment, 4, pp. 519-553.

Giulianno, G., Small, K.A. (1991). Subcenters in Los Angeles Region, Regional Science and Urban Economics, 21, 163-182.

Gordon, P, Richarson, H.W. (1996). Beyond Polycentricity. The Dispersed Metropolis, Los Angeles, 1970-1990, Journal of the American Planning Association, Vol 62, nº 3, pp. 289-295

Heikkila, E., Gordon, P., Kim, J.I., Peiser, B., Richardson, H.W. y Dale-Johnson, D. (1989). What happened to the CBD-Distance Gradient? Land values in a polycentric city, Environment and Planning A, 21, pp. 221-232.

Marmolejo, C., Roca, J. (2006). Hacia un modelo teórico del comportamiento espacial de las actividades de oficina. Scripta Nova. Revista electrónica de geografía y ciencias sociales. Barcelona: Universidad de Barcelona, 15 de julio de 2006, vol. X, núm. 217. McDonald, J.F. (1987). The Identification of Urban Employment Subcenters, Journal of Urban Economics, 21, pp. 242-258.

McDonald, J.F.; McMillen, D.P. (1990). Employment Subcenters and Land Values in a Polycentric Urban Area: The Case of Chicago, Environment and Planning A, 22, pp. 1561-1574.

McDonald, J.F; McMillen, D.P. (1998). "Land values, land use, and the first Chicago Zoning Ordinance, Journal of Real Estate Finance and Economics Vol. 16, no 2, pp. 135-150.

McDonald, J.F., McMillen, D.P. (2007). Urban economics and real estate: theory and policy, Blackwell, Malden MA, Boston USA. PP 640.

McMillen, D.P. (2003). Identifying Subcentres Using Contiguity Matrices, Urban Studies Vol. 40, no 3, pp. 57-69.

McMillen, D.P. (2001). "Non-Parametric Employment Subcenter Identification", Journal of Urban Economics, 50, pp. 448-473.

McMillen, D.P. (1996). "One Hundred Fifty Years of Land Values in Chicago: A Nonparametric Approach", Journal of Urban Economics, 40, pp. 100-124. 


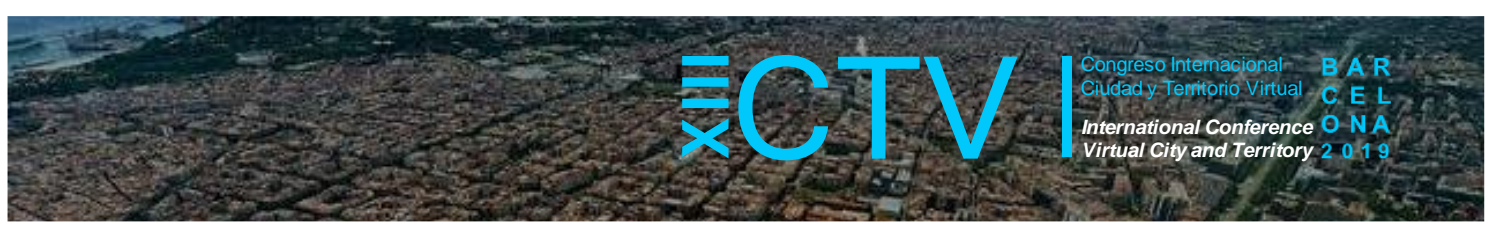

McMillen, D.P.; McDonald, J.F. (1997). "A Nonparametric Analysis of Employment Density in a Polycentric City", Journal of Regional Science, 37, pp. 591-612.

Muñiz, I, (2003). ¿Es Barcelona una ciudad policentrica?; Working Paper 03.09; Departament d'Economia Aplicada; UAB.

Muñiz, I; Galindo, A. (2005). Descentralisation, integration and polycentrism in Barcelona, Working paper,05.12 del departamento de Economia Aplicada Univerdidad Autónoma de Barcelona.

Muth, R. (1969). Cities and Housing. Chicago: University of Chicago. Chicago, Illinois, Estados Unidos.

Roca J. (1988) "La Estructura de valores urbanos un análisis teórico-empírico, 1era edición, Instituto de Estudios de Administración Local, Madrid.

Roca, J. Marmolejo, C; Moix, M; (2010). "Estructura Urbana y Policentrismo. Hacia una redefinición del concepto", Urban studies (Forthcoming)

Roca, J.; Moix, M. (2005). "Cap a una nova organizacio territorial de Catalunya" Research paper, 5-2004, Centro de Política de suelo y valoraciones, Universidad Politécnica de Cataluña.

Redfearn, C.L. (2007): "The Topography of Metropolitan Employment: Identifying Centers of Employment in a Polycentric Urban Area", Journal of Urban Economics, 61, pp. 519-561.

Shearmur, R.; Coffey, W.J. (2002). "A Tale of Four Cities: Intrametropolitan Employment Distribution in Toronto, Montreal, Vancouver, and Ottawa-Hull, 1981-1996", Environment and Planning A, 34, pp. 575-598.

Shearmur, R. (2018). The Millennial Urban Space-Economy: Dissolving Workplaces and the Delocalization. In M. Moos, D. Pfeiffer, \& T. Vinodrai, The Millennial City, Trends, Implications, and Prospects for Urban Planning and Policy (pp. 65-79). Oxford: Routledge.

Song, S. (1994). Modelling Worker Residence Distribution in the Los Angeles Region, Urban Studies 31, pp. 1533-1544.

White, M.J. (1976). Firm Suburbanization and Urban Subcenters. Journal of Urban Economics, 3, pp. 323-343. 